\title{
The functional verification of EGFR-CAR T-cells targeted to hypopharyngeal squamous cell carcinoma
}

This article was published in the following Dove Press journal: OncoTargets and Therapy

\author{
Yi-Han Dong' \\ Yi-Ming Ding ${ }^{2}$ \\ Wei Guo \\ Jun-Wei Huang ${ }^{2}$ \\ Zheng Yang ${ }^{2}$ \\ Yang Zhang ${ }^{2}$ \\ Xiao-Hong Chen ${ }^{2}$ \\ 'Department of Otolaryngology, \\ Daqing Oilfield General Hospital, \\ Daqing City I6300I, Heilongjiang \\ Province, People's Republic of China; \\ ${ }^{2}$ Department of Otolaryngology Head \\ and Neck Surgery, Beijing Tongren \\ Hospital, Capital Medical University, \\ 100730, Beijing, People's Republic \\ of China
}

Background: The aim of this study was to validate the antitumor function of EGFR-chimeric antigen T-cells (CART) targeted to FaDu cells, a hypopharyngeal squamous cell carcinoma cell line, and to provide a preclinical basis for the application of CART cell technology in hypopharyngeal squamous cell carcinoma.

Methods: Detection of cytokine secretions of EGFR-CAR T and CART-controls in the presence of target cells and nontarget cells as an indicator of CART cell activation. Detection of the cytotoxic effects of EGFR-CAR T on specific tumors in the presence of target cells was evaluated by LDH release and CART cell proliferation.

Results: The results showed that cytokine secretion increased significantly after EGFR-CAR T-cells were incubated with target cells, and EGFR-CAR T-cells has higher cytotoxic effect on target cells than the CART-control group. The target cell lysis rate was $52.66 \%$. The proliferation of EGFR-CAR T-cells in the presence of target cells was not distinctly observed.

Conclusion: In this study, we validated the antitumor function of EGFR-CAR T-cells targeted to the FaDu cell line and provided the foundation for application of the CART technique in the treatment of hypopharyngeal carcinoma.

Keywords: chimeric antigen receptor T-cells, epidermal growth factor receptor, hypopharyngeal neoplasm

\section{Introduction}

Hypopharyngeal carcinoma accounts for approximately $5 \%$ of head and neck cancers and mostly appears as squamous cell carcinomas. It is usually found at the pyriform sinus, though it is sometimes found at the posterior wall of the laryngeal pharynx and seldom found at the postcricoid area. ${ }^{1}$ Currently, the first-line treatment of hypopharyngeal carcinoma is a combined modality therapy, which includes surgery and preoperative or postoperative radiochemotherapy. ${ }^{2}$ Due to the critical biological characteristics of hypopharyngeal carcinoma, cervical lymph node metastasis is quite common in the disease and is difficult to detect; the 5-year survival rate of this cancer is only approximately $40 \%{ }^{3}$

Chimeric antigen receptor T-cells (CART) therapy is a type of adoptive cellular immunotherapy. The principle of CART is to collect the T-cells of an affected patient, introduce the coding gene of an antibody-like protein by genetic engineering technology into T-cells (such as an antibody to cancer cell antigen, cell receptor fragment, or T-cell proliferation stimulating factor that specifically binds tumor cell surface antigen), amplify the T-cells in vitro, and transfuse them into the patient to produce an antitumor effect. ${ }^{4}$ CART therapy does not rely on the MHC mechanism to identify and

\footnotetext{
Correspondence: Yang Zhang; Xiao-Hong Chen

Department of Otolaryngology, Beijing Tongren Hospital, Capital Medical University, No I Dongjiaominxiang Street, Dongcheng District, Beijing, People's Republic of China Tel +86 I33 I| 365369 ;

$+8613911071002$

Email zhangyangent@163.com; trchxh@I63.com
} 
act on tumor cells. CART can specifically target malignant cells and produce an accurate antitumor effect, thereby providing a novel therapeutic approach for refractory cancer. ${ }^{5}$ The most successful clinical application of CART therapy is in the treatment of hematologic malignancy, such as the use of CD19-CAR T-cells to treat B cell malignancies. The complete response rate in this application can be up to $70 \%{ }^{6}$ However, the application of CART therapy in the treatment of solid tumors is still at an exploratory stage. The major setback is determining a target for CART cells.

EGFR, one type of receptor tyrosine kinase (Tk), ${ }^{7}$ is highly expressed in several kinds of human malignancies, such as squamous cell carcinomas of the head and neck, colorectal carcinomas, non-small-cell lung cancer, breast cancer, malignant gliomas, and prostate cancer. ${ }^{8}$ EGFR is also overexpressed in the hypopharyngeal carcinoma FaDu cell line and plays an important role in the occurrence and development of these cells. ${ }^{9}$ In our previous study, we successfully constructed and verified EGFR-CAR T-cells. ${ }^{10}$ In this manuscript, we show the antitumor effects of EGFRCAR T-cells, which have the potential to serve as an immunotherapy in the treatment of hypopharyngeal squamous cell carcinoma.

\section{Materials and methods}

\section{The construction of EGFR-CAR T-cells}

\section{Reagents and instruments}

Ficoll-Paque PLUS (GE, Boston, MA, USA, cat\#17-1440-02), complete medium: TexMACS (Miltenyi Biotechnology, Bergisch Gladbach, Germany, cat \#170-076-309) + IL-2 (Miltenyi Biotechnology, cat\#130-097-748), PerCP5.5 antihuman CD3 (BD, Franklin Lakes, NJ, USA, cat \#552852), PE anti-human CD4 (BD, cat \#555347), APC anti-human CD8 (BD, cat \#555369), APC anti-human EGFR (Biolegend, San Diego, CA, USA, cat \#352906) FaDu (Minimum Essential Medium $+10 \%$ fetal bovine serum [FBS]), HCTRPMI 1640 (1640+10\% FBS), FACS buffer: PBS +2.5\% FBS, flow cytometry: Millipore Guava easyCyte HT (Merck Millipore, Burlington, MA, USA), $\mathrm{CO}_{2}$ incubator: ESCO MCO-20AIC (Ehime-ken, Japan), cell counter: Cellometer Auto 1000 (Nexcelom Bioscience LLC, Lawrence, MA, USA) were all used.

Peripheral blood mononuclear cells were isolated via the Ficoll density gradient centrifugation method from whole blood samples of healthy volunteers at the Department of Blood Transfusion, Beijing Tongren Hospital. This study was approved by the ethics committee of Beijing Tongren Hospital (ethics number TRECKY2014-027), and the healthy volunteers whose blood samples were used had provided written informed consent. The FaDu cell lines and HCT-116 cell lines were purchased from Cell Bank, Shanghai Institutes for Biological Sciences, Chinese Academy of Sciences (Shanghai, People's Republic of China).

\section{Methods}

After the peripheral blood mononuclear cells were isolated and amplified, the experimental group (EGFR CAR T) was treated with the EGFR two-generation CAR lentivirus, and the control group (CART Con) was treated with the control virus. After culture and amplification, cell type was determined by the following steps: FACS buffer was added to overhanging cells, and APC anti-human CD8, anti-human CD4, and PerCY5.5 anti-human CD3 were then added to cells. After incubation and washing, FACS buffer was added to overhanging cells, and FACS was used to detect fluorescence. The infection efficiency was measured as follows: the transfected CART cells were added to the FACS buffer of overhanging cells and APC anti-human EGFR was added to the FACS buffer after cleaning. FACS buffer was then added to the cell suspension, and finally, FACS was used to detect fluorescence. The EGFR expression level in FaDu cells and HCT-116 cells was detected by the methods described above. The specific methods are detailed in our previous study. ${ }^{10}$

\section{Detection of CART cytokine secretion Cytokine secretion}

We collected the target cells (FaDu cell line), washed the cells with dilute buffer, centrifuged at 1,000 rpm for 3 minutes, and then discarded the supernatant. Cells were resuspended with $2 \%$ FBS and then diluted to $1 \times 10^{6} / \mathrm{mL}$. Nontarget cells (HCT-116), EGFR CAR T, and CART-controls were treated in the same way. In 96-well plates, $100 \mu \mathrm{L}$ of target cells or nontarget cells and $100 \mu \mathrm{L}$ of EGFR CAR T or CARTcontrols were mixed in a 1:1 proportion and then cocultured for 20-24 hours at $37^{\circ} \mathrm{C}$ and $5 \% \mathrm{CO}_{2}$. Cells were centrifuged at 1,000 rpm for 3 minutes, and the supernatant was collected for cytokine detection.

\section{Cytokine detection}

To determine the number of cytokines and the number of samples to test, we calculated the number of cytokine specimens to detect as follows: (the number of samples to detect + the standard number + the negative control number $) \times 10 \mu \mathrm{L}$. This was calculated for each sample and the samples were mixed with a turbine concussion. The samples were diluted with a standard dilution taking $\mathrm{N}=1.5 \mathrm{~mL}$ EP tubes 
(where $\mathrm{N}=$ sample number + standard number + control number). Then, $50 \mu \mathrm{L}$ of prepared sample was added to each tube (standard, sample, or negative control), $50 \mu \mathrm{L}$ of the corresponding test agent (standard, sample, or negative control) was added, and $50 \mu \mathrm{L}$ of PE Detection Reagent was added to each tube. After fully mixing, samples were incubated at room temperature for 3 hours. Then, $1 \mathrm{~mL}$ of wash buffer was added to each tube, followed by centrifugation at $200 \mathrm{rpm}$ for 5 minutes. Wash buffer $(300 \mu \mathrm{L})$ was added to each tube and resuspended to overhanging precipitation of the tube. Samples were detected by flow cytometry. The result was analyzed by FCAP Array v3 from BD.

\section{Detection of CART cytotoxicity}

Target cells were collected and cleansed with a diluted buffer, centrifuged at 1,000 rpm for 3 minutes, and the supernatant was discarded. Cells were resuspended with RPMI 1640 with $2 \% \mathrm{FBS}$, and the cell density was adjusted to a final density of $1 \times 10^{5} / \mathrm{mL}$. The CART (effect cells) were treated in the same way, and the cell density was finally adjusted to $2 \times 10^{6} / \mathrm{mL}, 1 \times 10^{6} / \mathrm{mL}, 0.5 \times 10^{6} / \mathrm{mL}, 0.25 \times 10^{6} / \mathrm{mL}$, and $0.125 \times 10^{6} / \mathrm{mL}$. The cells were added to U-type bottom 96-well plates ( $200 \mu \mathrm{L}$ system). The grouping is shown in Table 1, E:T is 20:1, 15:1, 10:1, 5:1, 1:1. The cells were incubated at $37^{\circ} \mathrm{C}$ in $5 \% \mathrm{CO}_{2}$ for $4-6$ hours. At 45 minutes before the end of the experiment, $20 \mu \mathrm{L}$ of lysis buffer was added to the maximum-release group of target cells. Culture plates were centrifuged at 1,500 rpm for 5 minutes, and then $50 \mu \mathrm{L}$ of supernatant per well was transferred to new 96-well plates. Following this, $50 \mu \mathrm{L}$ of mixed substrate was added to each well, incubated at room temperature for 30 minutes, and $50 \mu \mathrm{L}$ of terminating solution was added. Absorption value was detected at $490 \mathrm{~nm}$, and LDH activity was detected in cell culture supernatant. The data were analyzed using Flow Jo 7.6.1 (FlowJo, LLC, Ashland, OR, USA).

Table I Experimental grouping of cytotoxicity detection of CART

\begin{tabular}{l|l}
\hline Group & System composition \\
\hline $\begin{array}{l}\text { Culture medium background } \\
\text { control }\end{array}$ & Culture medium $200 \mu \mathrm{L}$ \\
\hline Target cell self-release & $\begin{array}{l}\text { Culture medium } 100 \mu \mathrm{L} \text {, target cell } \\
100 \mu \mathrm{L}\end{array}$ \\
\hline $\begin{array}{l}\text { Cell release with different } \\
\text { concentration effects }\end{array}$ & $\begin{array}{l}\text { Culture medium } 100 \mu \mathrm{L}, 100 \mu \mathrm{L} \text { effect } \\
\text { cells of different concentrations }\end{array}$ \\
\hline Target cell maximum release & $\begin{array}{l}\text { Culture medium } 100 \mu \mathrm{L} \text { added } \\
\text { to target cell } 100 \mu \mathrm{L}\end{array}$ \\
\hline Different target ratio group & Target cell $100 \mu \mathrm{L}$, effect cell $100 \mu \mathrm{L}$ \\
\hline
\end{tabular}

Abbreviation: CART, chimeric antigen T-cells.

\section{Detection of CART cell proliferation in the presence of target cells}

Target cells were collected and cleansed with a dilute buffer, centrifuged at 1,000 rpm for 3 minutes, and the supernatant was discarded. Cells were resuspended with RPMI 1640 with $2 \% \mathrm{FBS}$, and the cell density was adjusted to a final density of $1 \times 10^{5} / \mathrm{mL}$. The CART (effect cells) were treated in the same way except for adjusting to a cell density of $2 \times 10^{5} / \mathrm{mL}$. In all, $1 \mathrm{~mL}$ of effector cells and target cells was added to 12 -well plates, incubated for 72 hours at $7^{\circ} \mathrm{C}$, and $5 \% \mathrm{CO}_{2}$. GFP-positive cells were detected by FACS.

\section{Results}

\section{The construction of EGFR-CAR T-cells}

The infection rate of EGFR-CAR T-cells was approximately $67.13 \%$, and the infection rate of the CART-control group was approximately $67.46 \%$. There was no significant difference in the distribution of cell subsets between the EGFR-CAR T and CART-control groups. T lymphocytes $\left(\mathrm{CD}^{+}\right)$accounted for $99.9 \%$ of the total cell population. Of the $\mathrm{T}$ lymphocytes, $\mathrm{T}$ effect or lymphocytes $\left(\mathrm{CD} 3^{+} \mathrm{CD} 8^{+}\right)$ accounted for approximately $25 \%$ of T-cells, and T assisted lymphocytes $\left(\mathrm{CD}^{+} \mathrm{CD}^{+}\right)$accounted for approximately $68 \%$. The expression of EGFR in EGFR-CAR $\mathrm{T}$ target cells and nontarget cells was detected. It was found that the HCT-116 cell lines had low expression of EGFR and that the FaDu cell lines highly expressed EGFR. These specific results have been detailed in our previous study. ${ }^{10}$

\section{Detection of CART cytokine secretion}

The cytokine secretion results of both EGFR-CAR $T$ and CART-controls in the presence of target cells and nontarget cells are presented in Figure 1. We found that, in the presence of target cells, the secretion of IL-2 in EGFR-CAR T-cells was detectable but lower than that of the CART-control group. The secretion of IL-6 in EGFR-CAR T-cells was higher than that of the control group, but the difference was minor. However, the secretion of IL-4, IL-10, TNF- $\alpha$, and IFN- $\gamma$ in EGFR-CAR T-cells was much higher than that of the CART-control group, which may be indicative of T-cell activity. The results show that, in the presence of target cells, EGFR-CAR T-cells have functional activity.

\section{Detection of CART cytotoxicity}

The results of the cell lysis effects of EGFR-CAR T-cells and the CART-control group on the target cells and nontarget cells are presented in Figure 2. In the CART-control group, 

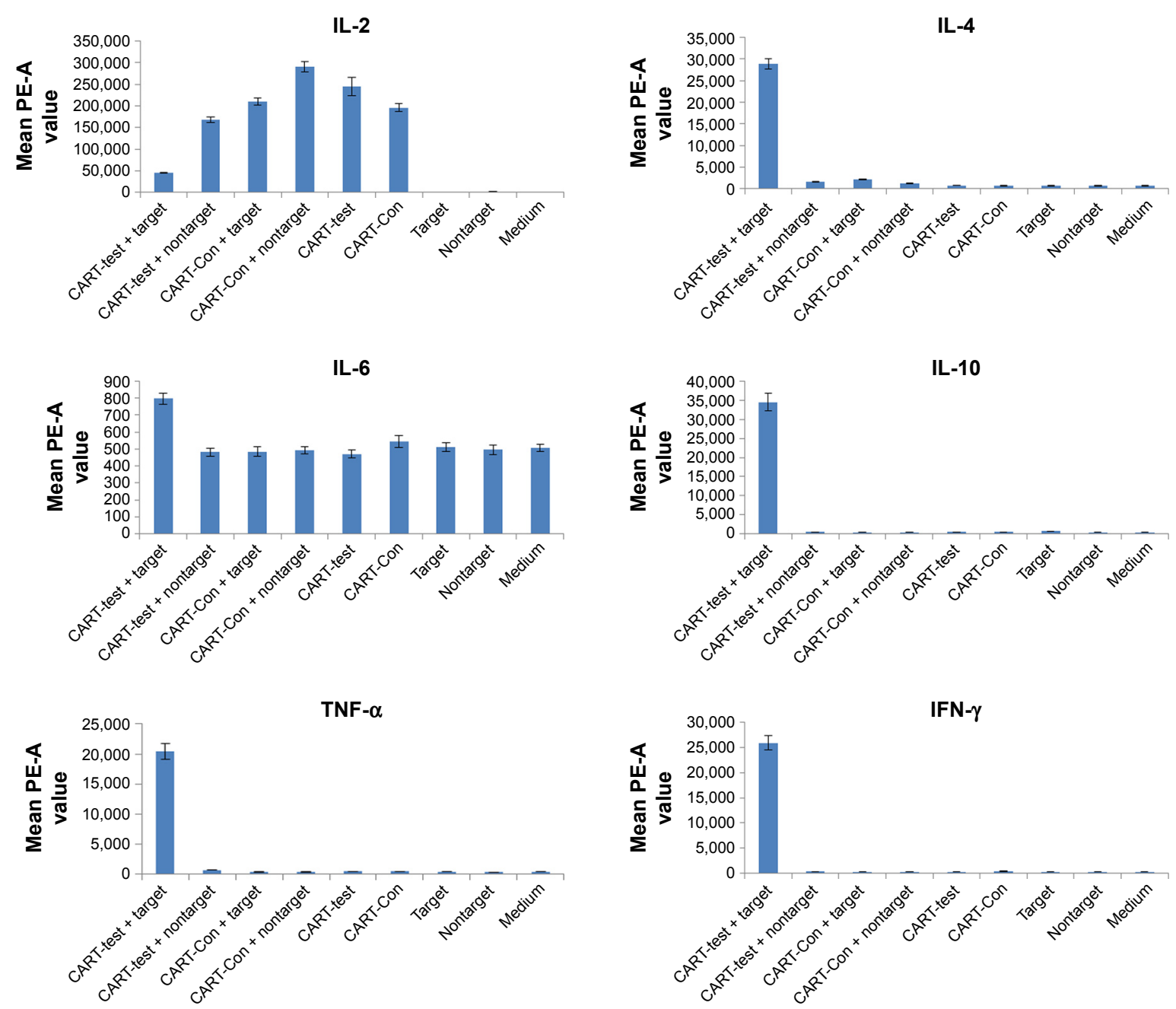

Figure I Cytokine secretion of CART-EGFR and CART-control groups. Abbreviations: CART, chimeric antigen T-cells; Con, control.

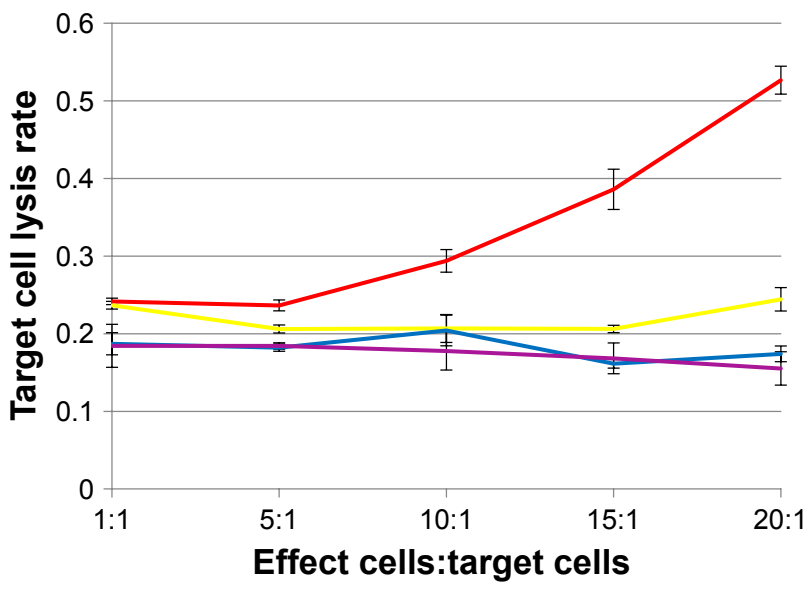

\begin{tabular}{|lr|}
\hline CART-test/target & CART-test/nontarget \\
CART-Con/target & CART-Con/nontarget \\
\hline
\end{tabular}

Figure 2 Cytotoxicity of CART-EGFR and CART-control groups on target and nontarget cells.

Abbreviations: CART, chimeric antigen T-cells; Con, control. as well as the EGFR-CAR T-cells with nontarget cells, the cell lysis rate was low and does not rise with the target cell ratio. However, in the EGFR-CAR T-cells with target cells group, the cell lysis rate was much higher than the other three groups and rose with the target cell ratio. After coculturing for 4 hours at a target cell ratio of 20:1, the target cell lysis rate of EGFR-CAR T-cells with target cells was as high as $52.66 \%$. Therefore, compared with the CART-control group, EGFR-CAR T-cells have an established cytotoxicity on target cells.

\section{The proliferation of CART cells in the presence of target cells}

The results of cell proliferation in EGFR-CAR T-cells and CART-control are shown in Figure 3. We found that at different ratios of CART cells and target cells, the difference in 

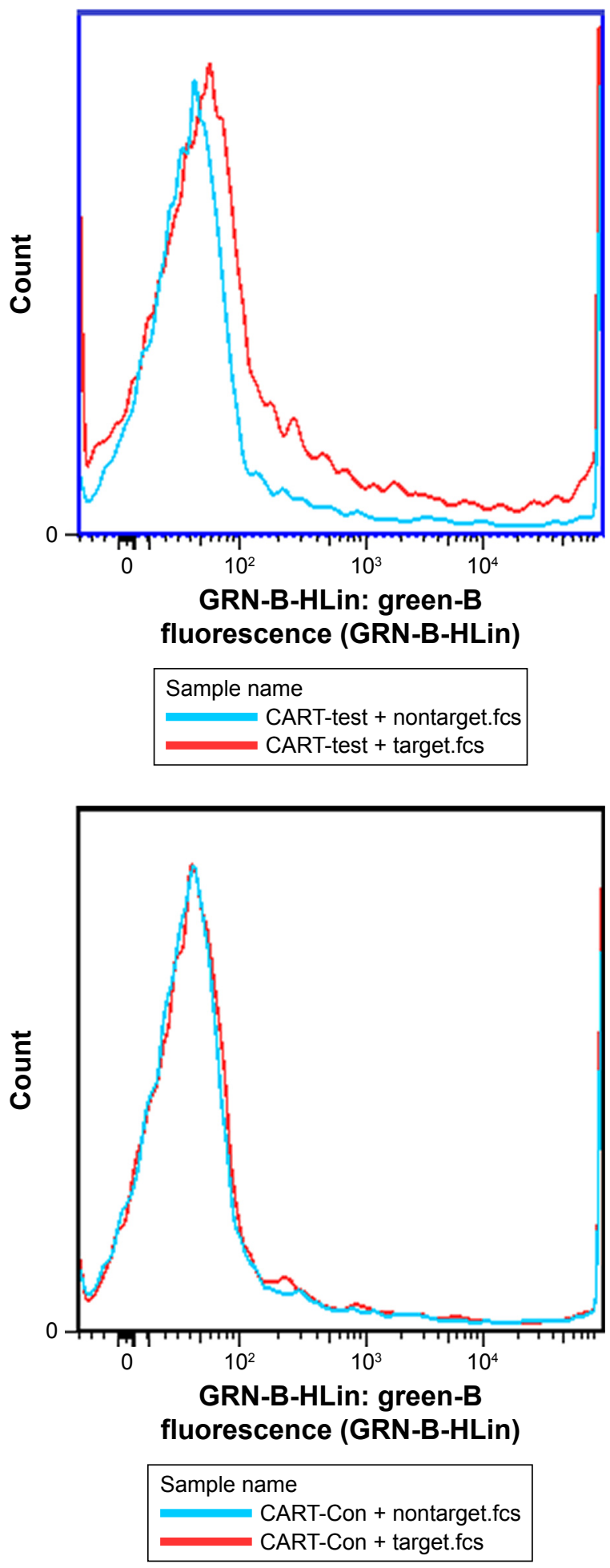

Figure 3 Detection of GFP cells in CART-EGFR and CART-control groups. Abbreviations: CART, chimeric antigen T-cells; Con, control.

proliferation of EGFR-CAR T-cells and CART control was not substantial. This finding indicates that the proliferation of CART cells would not be influenced by the EGFR-CAR $\mathrm{T}$-cells in the presence of T-cells.

\section{Discussion}

In this research, we utilized a previously constructed CART cell line targeted to EGFR on hypopharyngeal squamous cell carcinoma and studied the effects on a cytological level. By verifying the cytokine secretion and target cell lysis of EGFR-CAR T-cells in comparison to CART control in the presence of target cells and nontarget cells, we verified the antitumor effects of EGFR-CAR T-cells. The results showed that when exposed to target cells, EGFR-CAR T-cells secreted a large number of cytokines, such as IL-4, IL-6, IL-10, TNF- $\alpha$, and IFN- $\gamma$. These findings indicate that EGFR-CAR T-cells were in an activated state. Moreover, many target cells were lysed under the action of EGFR-CAR T-cells, indicating that EGFR-CAR T-cells have a strong tumor killing effect. Overall, it was shown that EGFR-CAR T-cells were activated and produced an antitumor effect through targeting EGFR in hypopharyngeal squamous cell carcinoma.

CART cell therapy has unique advantages in the treatment of hematological malignancies, especially in B cell malignancies. CD19 is specifically expressed in B cells, at all stages of development, differentiation, and malignancies, but is absent from hematopoietic stem cells and other cells. ${ }^{11}$ There are more than 20 clinical trials which are being carried out using CD19-CAR T-cells in the treatment of hematological malignancies, including chronic lymphoblastic leukemia, non-Hodgkin's lymphoma, and acute lymphoblastic leukemia. For patients with chronic lymphoblastic leukemia and non-Hodgkin's lymphoma, nearly half of the patients achieved complete remission, partial remission, or progression-free survival after receiving CD19-CAR T-cell treatment. ${ }^{12}$ For patients with acute lymphoblastic leukemia, the effect was more significant. ${ }^{13}$ Although there were many differences in terms of CAR structure, pretreatment regimen, dose of T-cells, and patient population, a number of clinical studies have reported high complete remission $(70 \%-90 \%) .{ }^{14}$ Hematological malignancies originate from plasma cells, such as in the case of multiple myeloma, but common CART cell targets on the surface of tumor cells are relatively absent in these cases. A study showed that B cell maturation antigen was specifically expressed on the surface of multiple myeloma cells and promoted their proliferation. ${ }^{15}$ Targets of CART cell are also difficult to identify in myeloid malignancies. In the current study, it was found that the expressions of CD33 and CD123 were significantly upregulated in myeloid malignancies. However, CD33 and CD123 are also expressed in normal hematopoietic stem cells, which means their targeting may lead to serious side effects. ${ }^{16,17}$ 
Endogenous tumor infiltrating T-cells have been considered a primary method to protect against cancer. ${ }^{18} \mathrm{CART}$ cells have similar antitumor effect as tumor infiltrating T-cells and can reach any organ of the body with blood circulation. Theoretically, the use of CART is one of the most effective methods to treat malignancy. Nevertheless, specific antigens on solid tumors are limited. Furthermore, targets for CART cells in solid malignancy therapy are also expressed at a much lower level than in normal tissues, which cause side effects. Currently, research on CART cells as a means of therapy is deficient. Among these studies, a promising result was reported in a preclinical model of GD2+ tumors treated with GD2-CAR T-cells. In a Phase I clinical trial in the treatment of GD2+ neuroblastoma, GD2-CAR T-cell therapy showed stronger antitumor effects and few side effects, only presenting as mild fever and mild to moderate pain due to tumor necrosis. ${ }^{19}$ In the clinical trials of CART cells targeting ovarian cancer, mesothelioma, and pancreatic cancer, mesothelin-targeted CART cells were efficient and had moderate side effects. ${ }^{20}$

EGFR, a member of the receptor Tk ErbB family, is widely distributed on the surface of most normal cells. The combination of EGFR and EGF stimulates tyrosine protein kinase action, causing a series of biochemical reactions that transmits the EGF signal to the nucleus and regulates the growth and differentiation of cells. Studies show that EGFR is overexpressed in many tumors, and the detection rate of EGFR in head and neck squamous cell carcinoma can reach $52.1 \%-98.3 \%{ }^{21}$ As Tk activity is necessary for EGFR signal transduction, Tk inhibitors are effective for targeting Tks in the treatment of tumors. Therefore, cancer therapy targeting EGFR and its mutants is widely studied. Monoclonal antibodies targeting EGFR have shown high efficacy in the treatment of several kinds of cancers, such as the use of gefitinib in lung cancer and cetuximab in colorectal cancer. Antibodies are the first-line treatment for patients with advanced nonresectable tumors. ${ }^{22}$ However, although EGFR monoclonal antibody can improve the overall survival rate to a certain extent in the treatment of advanced head and neck squamous cell carcinoma, the curative effect is not satisfactory, and the overall response rates were only $10 \%-13 \% .{ }^{23}$ Chemoradiation with high-dose cisplatin remains the mainstay of treatment for patients with locally advanced head and neck squamous cell carcinoma. ${ }^{24}$ As EGFR is highly expressed in head and neck squamous cell carcinoma, it can theoretically be used as a target in the construction of CART cells. ${ }^{25}$ CART cell therapy targeted to EGFR has been studied in cancers such as non-small-cell lung cancer and malignant gliomas. To date, no research has been done on head and neck squamous cell carcinoma. Therefore, our study provides the foundation for the application of CART cell technology in the treatment of head and neck squamous cell carcinoma.

\section{Conclusion}

Our study successfully constructed EGFR-CAR T-cells and is the first to verify its antitumor ability on EGFR + hypopharyngeal carcinoma at the cellular level. Although follow-up studies are required, our results strongly encourage development of EGFR-CAR T-cells therapy for hypopharyngeal carcinoma.

\section{Disclosure}

Yi-Han Dong and Yi-Ming Ding are the co-first authors. The authors report no conflicts of interest in this work.

\section{References}

1. Chan JY, Wei WI. Current management strategy of hypopharyngeal carcinoma. Auris Nasus Larynx. 2013;40(1):2-6.

2. Song J, Chang I, Chen Z, Kang M, Wang CY. Characterization of side populations in HNSCC: highly invasive, chemoresistant and abnormal Wnt signaling. PLoS One. 2010;5(7):e11456.

3. Chen SW, Yang SN, Liang JA, Lin FJ, Tsai MH. Prognostic impact of tumor volume in patients with stage III-IVA hypopharyngeal cancer without bulky lymph nodes treated with definitive concurrent chemoradiotherapy. Head Neck. 2009;31(6):709-716.

4. Rotolo A, Karadimitris A, Ruella M. Building upon the success of CART19: chimeric antigen receptor T cells for hematologic malignancies. Leuk Lymphoma. 2018;59(9):2040-2055.

5. Sadelain M, Brentjens R, Davila M, et al. Abstract CT102: Efficacy and toxicity management of $19-28 \mathrm{z}$ CAR T cell therapy in B cell acute lymphoblastic leukemia. Cancer Res. 2014;74(19 Supplement): CT102-CT102.

6. Dias A, Kenderian SJ, Westin GF, Litzow MR. Novel Therapeutic Strategies in Acute Lymphoblastic Leukemia. Curr Hematol Malig Rep. 2016;11(4):253-264.

7. Nogi H, Kobayashi T, Suzuki M, et al. EGFR as paradoxical predictor of chemosensitivity and outcome among triple-negative breast cancer. Oncol Rep. 2009;21(2):413-417.

8. Kobayashi S, Boggon TJ, Dayaram T, et al. EGFR mutation and resistance of non-small-cell lung cancer to gefitinib. N Engl J Med. 2005; 352(8):786-792.

9. Krstevska V, Stojkovski I, Zafirova-Ivanovska B, Crvenkova S. Prognostic factors in patients with advanced hypopharyngeal squamous cell carcinoma treated with concurrent chemoradiotherapy. J BUON. 2012;17(2):327-336.

10. Zhang Y, Ding YM, Zhong Q. Preliminary study on the construction of CAR-EGFR modified T cells (EGFR-CART) in hypopharyngeal squamous cell carcinoma. Chin Arch Otolaryngol Head Neck Surg. 2018;25(1):17-21.

11. Turtle CJ, Hanafi LA, Berger C, et al. Immunotherapy of non-Hodgkin's lymphoma with a defined ratio of $\mathrm{CD}^{+}$and $\mathrm{CD}^{+} \mathrm{CD} 19$-specific chimeric antigen receptor-modified T cells. Sci Transl Med. 2016; 8(355):355ra116.

12. Jena B, Dotti G, Cooper LJ. Redirecting T-cell specificity by introducing a tumor-specific chimeric antigen receptor. Blood. 2010;116(7): 1035-1044.

13. Sommermeyer D, Hill T, Shamah SM, et al. Fully human CD19-specific chimeric antigen receptors for T-cell therapy. Leukemia. 2017;31(10): 2191-2199. 
14. Liu J, Zhong JF, Zhang X, Zhang C. Allogeneic CD19-CAR-T cell infusion after allogeneic hematopoietic stem cell transplantation in B cell malignancies. J Hematol Oncol. 2017;10(1):35.

15. Bellucci R, Alyea EP, Chiaretti S, et al. Graft-versus-tumor response in patients with multiple myeloma is associated with antibody response to BCMA, a plasma-cell membrane receptor. Blood. 2005;105(10): 3945-3950.

16. Kenderian SS, Ruella M, Shestova O, et al. CD33-specific chimeric antigen receptor $T$ cells exhibit potent preclinical activity against human acute myeloid leukemia. Leukemia. 2015;29(8):1637-1647.

17. Mardiros A, Dos Santos C, Mcdonald T, et al. T cells expressing CD123specific chimeric antigen receptors exhibit specific cytolytic effector functions and antitumor effects against human acute myeloid leukemia. Blood. 2013;122(18):3138-3148.

18. Ganesan AP, Johansson M, Ruffell B, et al. Tumor-infiltrating regulatory $\mathrm{T}$ cells inhibit endogenous cytotoxic $\mathrm{T}$ cell responses to lung adenocarcinoma. J Immunol. 2013;191(4):2009-2017.

19. Louis CU, Savoldo B, Dotti G, et al. Antitumor activity and long-term fate of chimeric antigen receptor-positive $\mathrm{T}$ cells in patients with neuroblastoma. Blood. 2011;118(23):6050-6056.
20. Adusumilli PS, Cherkassky L, Villena-Vargas J, et al. Regional delivery of mesothelin-targeted CAR T cell therapy generates potent and long-lasting CD4-dependent tumor immunity. Sci Transl Med. 2014; 6(261):261ra151.

21. Juergens RA, Bratman SV, Tsao MS, et al. Biology and patterns of response to EGFR-inhibition in squamous cell cancers of the lung and head \& neck. Cancer Treat Rev. 2017;54:43-57.

22. Gerber DE, Choy H. Cetuximab in combination therapy: from bench to clinic. Cancer Metastasis Rev. 2010;29(1):171-180.

23. Vermorken JB, Herbst RS, Leon X, Amellal N, Baselga J. Overview of the efficacy of cetuximab in recurrent and/or metastatic squamous cell carcinoma of the head and neck in patients who previously failed platinum-based therapies. Cancer. 2008;112(12):2710-2719.

24. Magnes T, Egle A, Greil R, Melchardt T. Update on squamous cell carcinoma of the head and neck: ASCO annual meeting 2017. Memo. 2017;10(4):220-223.

25. Li H, Huang Y, Jiang DQ, et al. Antitumor activity of EGFR-specific CAR T cells against non-small-cell lung cancer cells in vitro and in mice. Cell Death Dis. 2018;9(2):177.
OncoTargets and Therapy

\section{Publish your work in this journal}

OncoTargets and Therapy is an international, peer-reviewed, open access journal focusing on the pathological basis of all cancers, potential targets for therapy and treatment protocols employed to improve the management of cancer patients. The journal also focuses on the impact of management programs and new therapeutic agents and protocols on

\section{Dovepress}

patient perspectives such as quality of life, adherence and satisfaction. The manuscript management system is completely online and includes a very quick and fair peer-review system, which is all easy to use. Visit http://www.dovepress.com/testimonials.php to read real quotes from published authors. 